\title{
SUSTENTABILIDAD Y VARIABILIDAD CLIMÁTICA: ACCIONES AGROECOLÓGICAS PARTICIPATIVAS DE ADAPTACIÓN Y RESILIENCIA SOCIOECOLÓGICA EN LA REGIÓN ALTO-ANDINA COLOMBIANA ${ }^{1}$
}

\author{
Álvaro Acevedo-Osorio ${ }^{2}$ \\ Arlex Angarita Leiton ${ }^{3}$ \\ Mónica Viviana León Durán ${ }^{4}$ \\ Karen Lorena Franco Quiroga ${ }^{5}$
}

Recibido el 17 de mayo de 2015, aprobado el 29 de octubre de 2015 y actualizado el 6 de diciembre de 2016

DOI: 10.17151/luaz.2017.44.2

\section{RESUMEN}

La variabilidad climática constituye en la actualidad uno de los principales factores de riesgo para la agricultura y la seguridad alimentaria mundial. Los ecosistemas más vulnerables en Colombia son los que se ubican en la zona alto-andina donde miles de familias campesinas se dedican a la agricultura en pequeña escala. Los programas enfocados a adaptar la agricultura a la variabilidad climática no se apoyan suficientemente en el conocimiento local ni motivan a las comunidades para la acción. Esta investigación propuso un análisis de la relación entre sustentabilidad y adaptación a la variabilidad climática, reconociendo de manera participativa los principales factores de riesgo para la sustentabilidad de la agricultura a partir de indicadores y analizando la efectividad de planes de mejora en la capacidad adaptativa de la agricultura alto-andina. Se desarrolló como un estudio de caso con 13 productores familiares vinculados a la Asociación Red Agroecológica Campesina de Subachoque, en 3 fases: a) creación de un marco de análisis sobre los principales factores de riesgo para la agricultura; b) evaluación de la sustentabilidad a partir de indicadores propios y c) implementación de planes para aumentar la capacidad adaptativa de la agricultura. La evaluación realizada en dos momentos diferentes demostró mejora en los 9 indicadores generados, de forma que la implementación de las acciones para mejorar la sustentabilidad influyeron positivamente sobre la capacidad de las fincas para adaptarse a los riesgos de la variabilidad climática. Las estrategias agroecológicas implementadas mejoraron la resiliencia socioecológica de los agroecosistemas manejados por agricultores familiares, aumentando la posibilidad de que respondan mejor ante cualquier escenario de cambio ambiental.

\section{PALABRAS CLAVE}

Sustentabilidad, adaptación de la agricultura, variabilidad climática, agroecología.

\section{SUSTENTABILITY AND CLIMATE VARIABILITY: PARTICIPATORY \\ AGROECOLOGYCAL ACTIONS TO ADAPTATION AND SOCIO-ECOLOGICAL REESILIENCE IN THE COLOMBIAN HIGH- ANDEAN REGION}

\begin{abstract}
Climate variability constitutes actually one of the main risks to agriculture and food security in the world. The most vulnerable ecosystems in Colombia are located in the high region of the Andeans where thousands of families farming lives from agriculture in small scale. The programs aimed to adapt the agriculture to climate variability do not take into account the local knowledge; neither motivates communities to action. This research proposed an analysis of the relationship between sustainability and
\end{abstract}


agricultural adaptation to climate variability in a participatory way, recognizing the main risk factors to agriculture sustainability using indicators and analyzing the effectiveness of improving plans to the agricultural adaptation capacity in the high-Andeans. It was developed as a study case with 13 farmers linked to the Agro-ecological Farming Net Association of Subachoque. 3 phases were developed: a) the creation of a framework regarding the main the risks factors to the agriculture; b) sustainability appraisal based on own indicators and c) implementation of plans to increase the agricultural adaptation capacity. The appraisal developed in 2 different moments showed improving in the 9 indicators suggesting that the implementation of actions to the sustainability of farming positively influenced on the farms capacity to face the risks of the climate variability. The agro-ecological strategies implemented, improving the socio-ecological resilience of agro-ecosystems managed by family farmers, increasing the possibility that they better responding to any scenario of environmental change.

\section{KEY WORDS}

Sustentability, agricultural adaptation, climate variability, agroecology.

\section{INTRODUCCIÓN}

El cambio climático global expresado en la variabilidad climática local y regional, constituye en la actualidad uno de los principales factores de riesgo para la agricultura y la seguridad alimentaria mundial. Diversos estudios predicen que la temperatura global se incrementará entre 1,4-5,8 grados centígrados, mientras que la precipitación decrecerá cerca de $20 \%$ en el próximo siglo afectando seriamente las regiones áridas y semiáridas del mundo; como consecuencia habrá una sustancial reducción de agua dulce y en la producción agrícola hacia finales del siglo XXI (Misra, 2014).

El Panel Intergubernamental sobre Cambio Climático (IPCC, 2007) estima que los principales riesgos asociados al cambio climático en los países andinos son el estrés de agua, la pérdida de biodiversidad y una mayor frecuencia de eventos hidrometereológicos extremos. En Colombia los ecosistemas más vulnerables son los que se ubican en las zonas altas de las cordilleras de los Andes (Boshell, 2010) donde miles de familias campesinas se dedican a una agricultura de pequeña escala.

Los efectos de la variabilidad climática serán especialmente severos en comunidades rurales de países menos desarrollados cuyos ingresos dependen fundamentalmente de la agricultura, además de estar técnica y financieramente menos dotados para adaptarse a situaciones cambiantes (Seaman, Sawdon, Acidri \& Petty, 2014). Se estima que el $47 \%$ de las áreas de economía campesina en Colombia podrían recibir alto y muy alto impacto por reducciones de lluvia en el período 2011 a 2040 (PNUD Colombia, 2011), situación especialmente delicada para el país si se tiene en cuenta que la agricultura campesina es responsable del $60 \%$ de la producción agrícola nacional con el $62 \%$ del área agrícola total; así mismo, los campesinos tienen el $70 \%$ de los predios dedicados a la ganadería y el 14\% del hato nacional (Forero, 2010).

Dos alternativas básicas se emplean para enfrentar los efectos del cambio climático que representan respuestas basadas en constantes ajustes socioambientales (Lahsen et al., 2010; Zimmerer, 2010): la adaptación busca, mediante una respuesta intuitiva (Meinke et al., 2009), fortalecer capacidades para que los agricultores convivan con las diversas manifestaciones de variabilidad climática; lluvias excesivas, sequías intensas 
o fuertes huracanes; por su parte, la mitigación tiene una perspectiva de largo plazo y busca disminuir las causas del cambio climático a través del rediseño de los sistemas productivos y sus correspondientes cadenas de distribución y consumo de alimentos para disminuir las tasas de emisión de Gases de Efecto Invernadero (GEI) y aumentar significativamente la captura del carbono (Zimmerer, 2010; Ríos, Miranda \& Vargas, 2011).

Los impactos de la variabilidad climática tienen características locales y, por lo tanto, no hay una estrategia de adaptación de la agricultura que sea única o aplicable en diversas escalas territoriales. En ese sentido, se debe evaluar la vulnerabilidad de cada sistema de producción en función de la amenaza, teniendo en cuenta que si el sistema de producción está bien adaptado ante la variabilidad natural del clima, hay una alta probabilidad de que responda bien ante cualquier escenario de cambio ambiental. Por consiguiente, en agricultura es más factible la reducción de la vulnerabilidad que la reducción de la amenaza (mitigación), para una adecuada adaptación ante la variabilidad climática (Boshel, 2010).

Los estudios relacionados a la variabilidad climática siguen dos tendencias generales, los que se enfocan en diagnosticar el impacto potencial y las consecuencias futuras evaluando distintas estrategias de adaptación mediante modelos de simulación (Crane, Roncoli \& Hoogenboom, 2011), y los que evalúan en campo diversas estrategias adaptativas para enfrentar las contingencias del clima y minimizar los riesgos afianzados en condiciones reales de las comunidades rurales.

Los estudios a partir de modelos de simulación no son adecuados para abordar los análisis de alternativas frente a la variabilidad climática, por cuanto aíslan los factores sociales que la pueden determinar, mientras que el enfoque de adaptación centrado en la comunidad (Crane et al., 2011) permite comprender la racionalidad de las comunidades rurales para hacer frente a la crisis climática que les afecta.

En general, los programas relacionados con la adaptación no toman en cuenta el punto de vista de los agricultores (Seidl, Morales, Arriola, \& Evangelista, 2011), siendo escasos los esquemas de evaluación que se apoyen en el conocimiento local o que motiven a las comunidades locales para la acción (Rogé \& Astier, 2013).

El presente estudio propuso un análisis de la relación entre sustentabilidad y adaptación a la variabilidad climática mediante un estudio de caso que buscó reconocer de manera participativa los principales factores de riesgo para la agricultura, evaluar la sustentabilidad y capacidad adaptativa a partir de indicadores y analizar la efectividad de planes de mejora en la capacidad adaptativa de la agricultura altoandina en una asociación de productores agroecológicos en Subachoque (Cundinamarca, Colombia).

\section{Agroecología, sustentabilidad y resiliencia socioecológica}

No existe una sola definición de agroecología. Se pueden identificar al menos dos grandes tendencias (Acevedo \& Angarita, 2013). La corriente ecologista la entiende como una ciencia creada para comprender, desde el punto de vista ecológico, las relaciones que se establecen entre los componentes de un agroecosistema, se centra en el estudio de las interacciones entre el medio natural y la producción agropecuaria (Altieri, 1995; Gliessman, 2002). La segunda corriente, basada en el enfoque de sustentabilidad, aborda la agroecología como un enfoque interdisciplinario para una agricultura sustentable; reconoce el aporte social y cultural de los agricultores tradicionales y se sustenta en el aporte del conocimiento tradicional preindustrial para la construcción de una agricultura que asegure tanto el cuidado ambiental como la 
justicia social y viabilidad económica (Guzmán, González \& Sevilla, 2000; SevillaGuzmán, 2006).

Diversos estudios plantean que la combinación de prácticas tradicionales de los sistemas de manejo y estrategias agroecológicas puede constituir una ruta factible para incrementar la productividad, la sostenibilidad y la resiliencia de los sistemas socioecológicos (Altieri \& Nicholls, 2013).

Por sistema socioecológico se entiende a aquel conformado en la interacción sociedad-naturaleza (Anderies, Janssen \& Ostrom, 2004). Un sistema socioecológico es sustentable cuando alcanza un estado socialmente deseable y ecológicamente posible y es insustentable si sufre un cambio que genera un estado socialmente indeseable prolongado o tiende a agotar la capacidad de carga de los ecosistemas de los que depende (Salas-Zapata, Ríos-Osorio \& Álvarez-Del Castillo, 2012). La capacidad adaptativa en un sistema socioecológico implica un balance o ajuste entre las actividades humanas y las dinámicas de los ecosistemas de manera que no se generen transformaciones que lleven al sufrimiento humano prolongado (Anderies et al., 2004; Salas-Zapata et al., 2012). En tanto enfoque metodológico y conceptual para alcanzar una forma de agricultura sustentable, la agroecología propende por mejorar la resiliencia socioecológica en los agroecosistemas (Ríos-Osorio, Salas-Zapata \& Espinosa-Alzate, 2013).

La resiliencia se refiere la capacidad del sistema de retornar a un estado de equilibrio o mantener el potencial productivo después de sufrir perturbaciones graves (Masera, Astier \& López-Ridaura, 1999); por ejemplo, perturbaciones ambientales como huracanes, incendios o inundaciones; perturbaciones económicas, como caídas drásticas de los precios del mercado o pérdida de mercados. Un sistemas socioecológico puede sobreponerse de dichas alteraciones y encontrar diferentes puntos de equilibrio reorganizándose a partir de cambios adaptativos (Ríos-Osorio et al., 2013).

Si bien las estrategias agroecológicas aumentan la resiliencia ecológica de los sistemas agrícolas, estas por sí solas no son suficientes para alcanzar la sustentabilidad; se hace necesario potenciar al mismo tiempo la resiliencia social, es decir, la capacidad de las comunidades para adaptarse frente a alteraciones causantes de estrés, sean sociales, políticas o ambientales (Altieri \& Nicholls, 2013).

\section{METODOLOGÍA}

El estudio fue desarrollado con 13 productores familiares vinculados a la Asociación Red Agroecológica Campesina (ARAC) de Subachoque, Cundinamarca, localizada en la región alto-andina colombiana a $2800 \mathrm{msnm}$ de altura, con $13,5^{\circ} \mathrm{C}$ de temperatura y una precipitación de $865 \mathrm{~mm}$ en promedio, zona de vida clasificada como Bosque Húmedo Montano (Holdrige, 1987).

La ARAC busca el buen vivir de sus integrantes mediante la aplicación de prácticas agroecológicas de producción, así como estrategias que fortalezcan sus redes familiares y comunitarias en armonía con el medio ambiente (Acevedo \& Angarita, 2013). Los agricultores asociados a la ARAC se dedican hace 3 años a la producción agroecológica de hortalizas, frutos y derivados lácteos para su mercadeo en Bogotá, ubicado a 45 kilómetros de distancia (Tabla 1). 
Tabla 1. Fincas de la Asociación Red Agroecológica Campesina de Subachoque

\begin{tabular}{|l|l|c|c|l|}
\hline$N^{\circ}$ & Finca & Area (ha) & $\begin{array}{c}\text { Altitud } \\
(\mathrm{msnm})\end{array}$ & Principales actividades productivas \\
\hline 1 & Serranias & 52 & 2643 & Hortalizas. Ganadería: leche y crías. \\
\hline 2 & El Porvenir & 1,4 & 2677 & Hortalizas. Miel de abejas. Huevos. \\
\hline 3 & Santa Otilia & 0,32 & 2681 & Hortalizas. Producción láctea (yogur). \\
\hline 4 & Las Hortensias & 0,64 & 2701 & Horticultura. \\
\hline 5 & $\begin{array}{l}\text { Campo } \\
\text { Hermoso }\end{array}$ & 0,08 & 2814 & Horticultura. Fruticultura. \\
\hline 7 & Ll Tablón & 0,32 & 2722 & Hortalizas. Frutas. \\
\hline 8 & Alcare & 0,32 & 2728 & $\begin{array}{l}\text { Agroindustria láctea (yogur, arequipe, } \\
\text { quesos). }\end{array}$ \\
\hline 9 & Buena Vista & 0,43 & 2740 & Horticultura. \\
\hline 10 & San Luis & 0,61 & 2675 & $\begin{array}{l}\text { Hortalizas. Agroindustria láctea (yogur, } \\
\text { quesos). }\end{array}$ \\
\hline 11 & La Acacia & 1,92 & 2958 & Hortalizas. Frutas. Papa. \\
\hline 12 & Almaguer & 0,13 & 2923 & Hortalizas. \\
\hline 13 & Los Laureles & 0,95 & 2784 & Hortalizas. \\
\hline
\end{tabular}

Fuente: los autores.

La investigación se desarrolló como estudio de caso en 3 fases: a) creación de un marco de análisis sobre los principales factores de riesgo para la agricultura (2012); b) evaluación de la sustentabilidad (y capacidad adaptativa) a partir de indicadores propios (2013) y c) implementación de plan de mejoras de la capacidad adaptativa de la agricultura (2013-2014).

Esta investigación dio continuidad a una anterior desarrollada por los investigadores en la misma asociación (Acevedo \& Angarita, 2013). El procedimiento metodológico se basó en las metodologías de marcos de análisis FESLM de la FAO (Smyth \& Dumanski, 1993); el marco teórico para la definición de indicadores de sustentabilidad del IICA-GTZ (Hünnemeyer, De Camino \& Müller, 1997) y el MESMIS creado por el grupo GIRA (Masera et al., 1999).

A partir de la consideración de que tanto la vulnerabilidad como las respuestas adaptativas están vinculadas a múltiples factores socioeconómicos, políticoinstitucionales, tecnológicos o ambientales y no solo a la variabilidad climática (Altieri, 2013), el marco de análisis y los indicadores construidos en el presente estudio tomaron como referencia la sustentabilidad y capacidad adaptativa como los referentes conceptuales.

Los indicadores propuestos fueron de tipo cuali-cuantitativo y para la evaluación fue construida una escala a partir de Lovell et al. (2010) que estandariza las variables asignando una valoración entre +2 y -2 para determinar si la contribución del indicador a la sustentabilidad y adaptabilidad del agroecosistema es positiva o negativa para la característica que está describiendo. 


\section{RESULTADOS Y DISCUSIÓN}

El marco de análisis para detectar riesgos para la sustentabilidad y adaptabilidad de los sistemas productivos de la ARAC ordenó los riesgos percibidos por los propios agricultores en una matriz de doble entrada con 5 dimensiones y 5 atributos del sistema productivo (Tabla 2). Los principales riesgos señalados en lo productivo se refieren a la baja productividad de las fincas, el uso de agroquímicos que aún persiste en varias fincas a pesar del cometido organizacional del no uso de estos insumos, y la debilidad en la planificación de la producción que hace frágil la presencia en los mercados de manera permanente.

En lo ambiental se destacan riesgos referidos a la calidad de los suelos, tanto por su baja fertilidad, sus bajos contenidos de materia orgánica como por los procesos erosivos que reducen la producción. La pérdida de semillas propias y alta dependencia de semillas certificadas del mercado convencional para la producción hortícola así como la escasez del recurso agua para la producción agropecuaria.

Los riesgos socioculturales se centran en bajo nivel de autosuficiencia alimentaria por una producción más enfocada al mercado, baja participación en las actividades de la asociación por parte de algunos de sus miembros, poca capacitación e investigación para la innovación productiva y baja incidencia ante consumidores externos. 
Tabla 2. Marco de análisis para detectar riesgos para la sustentabilidad y adaptabilidad de los sistemas productivos de la Red Agroecológica Campesina de Subachoque

\begin{tabular}{|c|c|c|c|c|c|}
\hline $\begin{array}{l}\text { Atributo } \\
\text { sistema }\end{array}$ & $\begin{array}{l}\text { Dimensión } \\
\text { productiva }\end{array}$ & $\begin{array}{l}\text { Dimensión } \\
\text { ambiental }\end{array}$ & $\begin{array}{l}\text { Dimensión } \\
\text { social }\end{array}$ & $\begin{array}{l}\text { Dimensión } \\
\text { cultural }\end{array}$ & $\begin{array}{l}\text { Dimensión } \\
\text { económica }\end{array}$ \\
\hline $\begin{array}{l}\text { Productividad } \\
\text { Capacidad del } \\
\text { agroecosistema de } \\
\text { producir bienes y } \\
\text { servicios para el } \\
\text { autoconsumo, } \\
\text { intercambio } \\
\text { mercado. }\end{array}$ & $\begin{array}{l}\text { Ganadería de } \\
\text { tipo extensivo. } \\
\text { Baja } \\
\text { productividad } \\
\text { agropecuaria. }\end{array}$ & \multirow{2}{*}{$\begin{array}{l}\text { Pérdida de } \\
\text { semillas } \\
\text { propias. } \\
\text { Reducción de } \\
\text { coberturas } \\
\text { vegetales } \\
\text { nativas. } \\
\text { Baja fertilidad } \\
\text { de los suelos. } \\
\text { Disminución } \\
\text { de } \\
\text { contenidos de } \\
\text { materia } \\
\text { orgánica. } \\
\text { Baja } \\
\text { disponibilidad } \\
\text { de agua para } \\
\text { la agricultura. }\end{array}$} & \multicolumn{2}{|c|}{$\begin{array}{l}\text { Poca producción para el } \\
\text { autoconsumo. }\end{array}$} & $\begin{array}{l}\text { Oferta no } \\
\text { constante } \\
\text { de } \\
\text { productos } \\
\text { para el } \\
\text { mercado. } \\
\text { Baja } \\
\text { rentabilidad. }\end{array}$ \\
\hline $\begin{array}{l}\text { Resiliencia } \\
\text { Capacidad de } \\
\text { actuar frente los } \\
\text { riesgos, } \\
\text { adaptándose y } \\
\text { disminuyendo la } \\
\text { vulnerabilidad. } \\
\text { Adaptabilidad } \\
\text { Capacidad de } \\
\text { encontrar nuevos } \\
\text { niveles de } \\
\text { estabilidadantelos } \\
\text { cambios. }\end{array}$ & $\begin{array}{l}\text { Persistencia } \\
\text { en del uso de } \\
\text { algunos } \\
\text { agroquímicos. }\end{array}$ & & \multicolumn{2}{|c|}{$\begin{array}{l}\text { Poca participación de } \\
\text { algunos miembros de la } \\
\text { ARAC en decisiones que } \\
\text { les implica. }\end{array}$} & \multirow[t]{2}{*}{$\begin{array}{l}\text { Mercado } \\
\text { escaso } \\
\text { hacia } \\
\text { consumidor } \\
\text { es } \\
\text { consciente. }\end{array}$} \\
\hline $\begin{array}{l}\text { Equidad } \\
\text { Posibilidad de } \\
\text { decidir de manera } \\
\text { equitativa sobre el } \\
\text { manejo y disfrutar }\end{array}$ & $\begin{array}{l}\text { Falta de } \\
\text { coordinación } \\
\text { de la } \\
\text { producción }\end{array}$ & & \multicolumn{2}{|c|}{$\begin{array}{l}\text { Falta de oportunidades } \\
\text { para el intercambio de } \\
\text { conocimientos. }\end{array}$} & \\
\hline
\end{tabular}




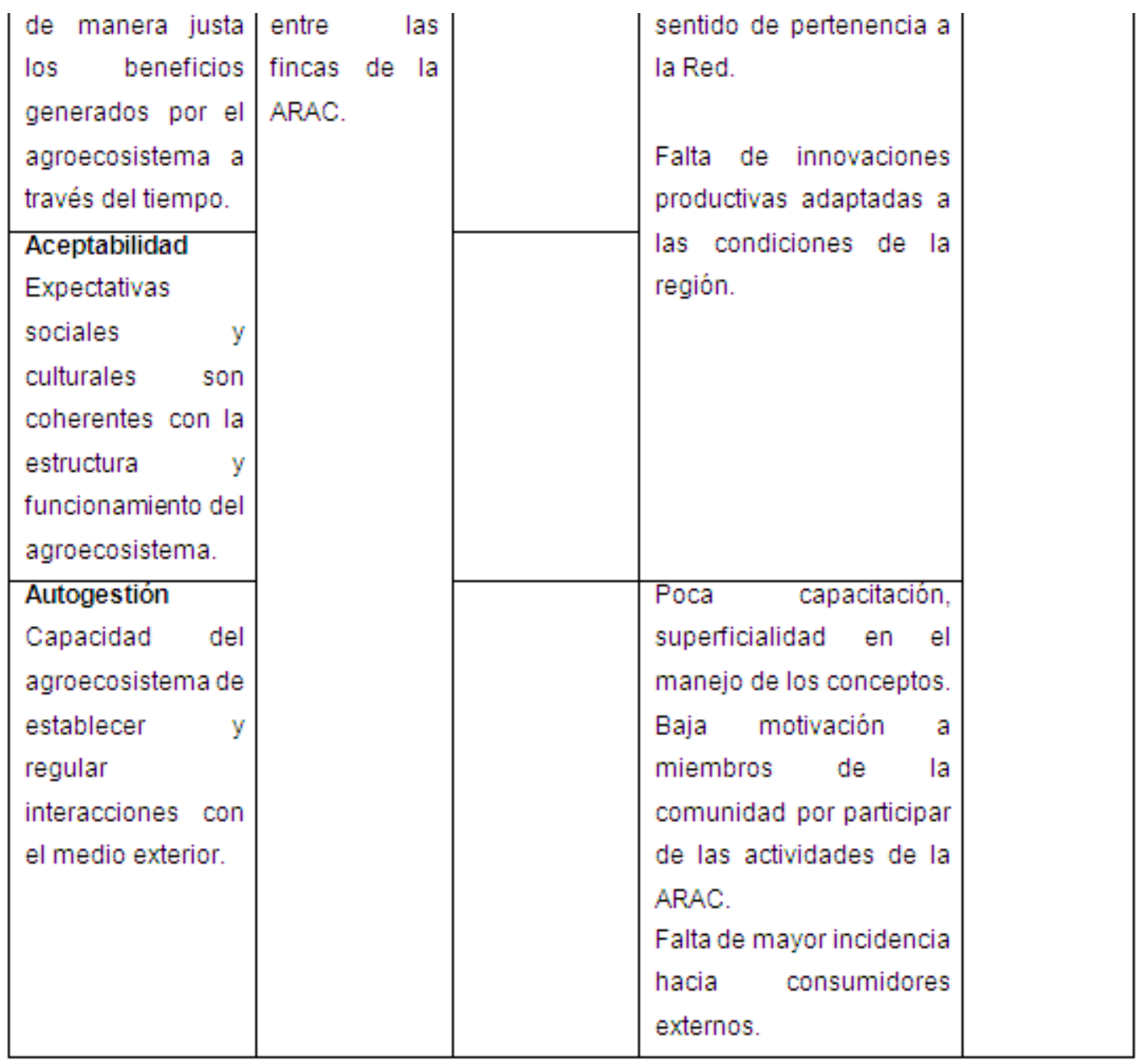

Fuente: los autores [a partir de Conway (1994) y Masera et al. (1999)].

Finalmente, en la dimensión financiera los agricultores resaltan como los principales riesgos la baja rentabilidad de su actividad productiva, presencia no constante en el mercado con los productos ofertados por la deficiencia en la planificación de las siembras de manera coordinada entre los asociados, así como la necesidad de ampliar el mercado para algunos de estos productos más abundantes.

La totalidad de dimensiones y atributos representadas en la matriz, quedaron relacionados en uno o varios de los riesgos mencionados, se percibe cómo unos riesgos están relacionados con otros, por ejemplo la baja fertilidad del suelo tiene incidencia en la baja productividad y baja rentabilidad; la dificultad en la planificación de la producción incide en la oferta no permanente de productos para el mercado, o la poca investigación e innovación tecnológica en la persistencia del uso de algunos agroquímicos. También, es importante resaltar que los riesgos no son diagnosticados solo para una dimensión, sino que además se entretejen en las cinco dimensiones del estudio, corroborando la necesidad de que estos análisis se hagan desde un enfoque complejo y multidimensional ya que la vulnerabilidad de la agricultura depende tanto del desarrollo del capital natural como del social (Altieri \& Nicholls, 2013).

La construcción del marco de análisis para detectar riesgos para la sustentabilidad y adaptabilidad de los sistemas productivos constituye sin duda el paso más importante del proceso metodológico participativo; un marco de análisis puede ayudar a definir los 
mayores riesgos para que los propios agricultores puedan trabajar sobre ellos de manera prioritaria, así, los conceptos de sustentabilidad y adaptabilidad se construyen a nivel local desde los puntos de vista, aspiraciones y necesidades de los actores locales; esto ratifica la idea de que es imprescindible construir marcos de análisis específicos para cada contexto socioeconómico específico (Kumaraswamy, 2012).

Aunque muchas metodologías de evaluación de sustentabilidad se basan en diversos indicadores sugeridos al azar, muchas veces por actores externos a la comunidad; evaluar el estado del sistema productivo implica mucho más que construir listados de indicadores (Masera et al., 1999); en tal sentido, cobra importancia el uso de marcos de análisis (Smyth \& Dumanski, 1993; Hünnemeyer et al., 1997; Masera et al., 1999) como sustento para la construcción de indicadores coherentes.

Con la priorización de los riesgos detectados por los agricultores se determinó un conjunto de 9 indicadores apropiados a las condiciones de la agricultura practicada por socios de la ARAC. Este conjunto de indicadores representa los aspectos más relevantes para mejorar la sustentabilidad y adaptabilidad de los sistemas de manejo, reduciendo la vulnerabilidad frente a la variabilidad climática y otros factores que pudieran alterar la estabilidad del sistema productivo.

La Tabla 3 muestra los resultados de dos evaluaciones de los sistemas productivos a partir de los 9 indicadores realizados. Los valores positivos denotan indicadores que aportan a la sustentabilidad y adaptabilidad de la agricultura, mientras que los valores negativos indican afectación a las mismas. A partir de los resultados de la primera evaluación, se adelantaron acciones en cada finca que condujeran a un mejor desempeño de los indicadores para las evaluaciones posteriores; la segunda evaluación da cuenta de las variaciones generadas por la implementación de los planes de acción en cada finca en un lapso de un año.

El manejo del agua constituye una de las más importantes acciones adaptativas para enfrentar riesgos del cambio climático, especialmente cuando se trata de sequías prolongadas. En el caso de Subachoque esta condición es especialmente importante dada la baja precipitación y la necesidad permanente de irrigación a cultivos hortícolas que son altamente dependientes de humedad superficial. El indicador pasó de un estado de afectación a la sustentabilidad y adaptabilidad del sistema a uno de contribución leve. Las acciones desarrolladas para su mejoramiento incluyen sistemas de irrigación en pequeña escala, adecuación de reservorios de agua y descontaminación de aguas grises.

La transición del uso de semillas certificadas a semillas locales adaptadas a las condiciones de la región, constituye uno de los principales obstáculos para la implementación de la propuesta agroecológica en la ARAC. Un cambio importante se ha generado con la introducción a la rutina productiva de especies mejor adaptadas como el yacón (Smallanthus sonchifolius) y un proceso de adaptación de semillas que anteriormente no se producian en la zona como cilantro (Coriandrum sativum), papa (Solanum tuberosum), espinaca (Spinacia oleracea) y algunas lechugas (Lactuca sativa). Aunque la dependencia de semillas externas sigue siendo predominante, este fue uno de los indicadores que marcó una mejora estadísticamente representativa respecto a la primera evaluación. 
Tabla 3. Resultados de dos evaluaciones de sustentabilidad y adaptabilidad de la agricultura en 13 sistemas productivos de la ARAC a partir de indicadores locales

\begin{tabular}{|c|c|c|c|c|}
\hline Indicador & Concepto & $\begin{array}{c}\text { Promedio } \\
\text { evaluación } \\
1 \\
(2013)\end{array}$ & $\begin{array}{c}\text { Promedio } \\
\text { evaluación } \\
2 \\
(2014)\end{array}$ & $\begin{array}{c}\text { Diferencia } \\
\text { evaluaciones } \\
1 \text { y } 2\end{array}$ \\
\hline $\begin{array}{l}\text { Conservación } \\
\text { de agua }\end{array}$ & $\begin{array}{l}\text { Nivel de preservación de fuentes, } \\
\text { captación de agua lluvia, } \\
\text { almacenamiento, riego eficiente y } \\
\text { reciclaje dentro del sistema } \\
\text { productivo }\end{array}$ & $-0,08$ & 0,42 & 0,50 \\
\hline $\begin{array}{l}\text { Producción } \\
\text { de semillas } \\
\text { propias }\end{array}$ & $\begin{array}{l}\text { Capacidad de la finca para } \\
\text { obtener cultivos a partir de } \\
\text { semillas orgánicas obtenidas en } \\
\text { la propia zona }\end{array}$ & $-0,27$ & 0,73 & $1,00^{*}$ \\
\hline Arborización & $\begin{array}{l}\text { Integración de árboles nativos al } \\
\text { sistema productivo }\end{array}$ & 0,81 & 1,27 & 0,46 \\
\hline $\begin{array}{l}\text { Estabilidad y } \\
\text { fertilidad del } \\
\text { suelo }\end{array}$ & $\begin{array}{l}\text { Aplicación de técnicas } \\
\text { agroecológicas que mejoran la } \\
\text { estabilidad y fertilidad del suelo }\end{array}$ & 0,04 & 0,96 & $0,92^{*}$ \\
\hline Rentabilidad & $\begin{array}{l}\text { Nivel de rentabilidad medida a } \\
\text { partir del índice de relación } \\
\text { beneficio/costo }\end{array}$ & 0,23 & 0,77 & $0,54^{*}$ \\
\hline $\begin{array}{l}\text { Participación } \\
\text { en red }\end{array}$ & $\begin{array}{l}\text { Grado de participación de los } \\
\text { miembros de la red contribuyendo } \\
\text { a su fortalecimiento }\end{array}$ & 0,46 & 1,15 & 0,69 \\
\hline $\begin{array}{l}\text { Investigación } \\
\text { campesina }\end{array}$ & $\begin{array}{l}\text { Desarrollo de investigaciones e } \\
\text { intercambio de conocimientos }\end{array}$ & $-0,77$ & 0,23 & $1,00^{*}$ \\
\hline $\begin{array}{l}\text { Planificación } \\
\text { de la finca }\end{array}$ & $\begin{array}{l}\text { Grado de planificación de } \\
\text { acuerdo con la capacidad } \\
\text { ecológica y productiva de la } \\
\text { misma }\end{array}$ & $-0,15$ & 0,81 & $0,96^{*}$ \\
\hline $\begin{array}{l}\text { Mercadeo } \\
\text { justo }\end{array}$ & $\begin{array}{l}\text { Mantenimiento de relaciones } \\
\text { campo-ciudad con beneficios } \\
\text { mutuos para el productor y el } \\
\text { consumidor }\end{array}$ & 0,46 & 1,08 & $0,62^{*}$ \\
\hline
\end{tabular}

* Diferencia estadísticamente significativa para $p<0,05$.

$$
\text { Fuente: los autores. }
$$

La arborización constituye una de las propuestas importantes para fortalecer la biodiversidad funcional de los sistemas de cultivo (Altieri \& Nicholls, 2007) en las fincas 
de la ARAC. Especies como el trompeto (Bocconia frutescens), acacia negra (Gleditsia triacanthos), arboloco (Smallanthus pyramidalis), aliso (Alnus acuminata), sauco (Sambucus nigra), entre otras propios de la zona alto-andina, permiten generar interacciones importantes entre los diversos componentes del agroecosistema incentivándose servicios ambientales importantes como la activación de la biología del suelo, el ciclo de nutrientes, la acción natural de predadores sobre parásitos de los cultivos, entre otras. El interés por la arborización está fundamentado en la conveniencia de complejizar la estructura del agroecosistema ya que una estructura simplificada lo hace más vulnerable a un evento de variación climática (Altieri \& Nicholls, 2007; Rogé \& Astier, 2013).

Aunque la arborización fue propuesta en este estudio como indicador adaptativo al cambio climático, futuros estudios deben integrar el análisis de la mitigación al cambio climático que genera esta acción por la captura de carbono tanto en la materia orgánica que se almacena en el suelo como en la masa arbórea (Hernández et al., 2011); el análisis debería contabilizar la cantidad de carbono total que estos sistemas agroecológicos diversificados pueden capturar y almacenar por largos períodos de tiempo, reduciendo la cantidad de carbono liberado a la atmósfera causante del calentamiento global (Molinet, Álvarez, González, Damas \& Ruiz, 2011).

En cuanto al indicador de estabilidad y fertilidad del suelo, los agricultores dieron prioridad a las prácticas dirigidas a conservar el suelo y mejorar su fertilidad previendo la erosión. Las acciones adelantadas incluyeron capacitación en campo e implementación de prácticas como conservación de suelos de ladera, uso del agronivel para trazos en curvas a nivel, adecuación de terrazas, labranza mínima y labranza cero, elaboración de abonos orgánicos tipo compostaje y cultivos de microrganismos locales para favorecer el proceso de descomposición aeróbica de la materia orgánica; todas estas prácticas de fácil comprensión y aplicación por parte de los agricultores.

El indicador de rentabilidad demostró un incremento, resultado del efecto sinérgico de los indicadores ya analizados que condujeron en su totalidad a un mejor desempeño ambiental y productivo del sistema (resiliencia ecológica); sin embargo, la rentabilidad no hubiera mejorado sin una mejora en la resiliencia social como se detallará en los indicadores siguientes.

La participación y trabajo en red constituyen indicadores enfocados hacia la resiliencia social (Altieri \& Nicholls, 2013). La ARAC fue creada por iniciativa de los propios agricultores que, interesados en generar una mejor relación con la naturaleza así como mejores condiciones de vida para sus familias y la comunidad, iniciaron un proceso organizativo que se ha mantenido por más de 3 años. Parte de su interés es un liderazgo compartido que asegure la continuidad de su misión organizativa a futuro, prioridad que queda expresada en este indicador que promueve la participación activa y genuina de todos los asociados en las actividades productivas, formativas, de comercio e integración que promueve la ARAC. Esta resiliencia social expresada en el trabajo constante por fortalecer su organización, contribuye al mismo tiempo con la resiliencia de los agroecosistemas (Altieri \& Nicholls, 2013) en el sentido de que tanto los análisis de riesgos como la generación de mejores estrategias adaptativas, se facilitan y fortalecen en el trabajo colectivo.

La investigación local constituye uno de los efectos importantes del trabajo en red. Para los socios de la ARAC investigar significa poner los conocimientos de cada actor al servicio de los demás; construir juntos mejores alternativas productivas frente a los problemas que detectan e innovar juntos a partir de sus propias experiencias. Diversas iniciativas en investigación campesina para la adaptación de la agricultura a la variabilidad climática han demostrado su efectividad para el diseño, construcción, 
implementación y evaluación de tecnologías locales mediante metodologías participativas. En Cuba, los Centros Locales de Innovación Agropecuaria constituyen una de estas iniciativas a escala local que integran agricultores y técnicos trabajando juntos para enfrentar la vulnerabilidad de la agricultura (Miranda, Sánchez, Sánchez, Lamela \& Álvarez, 2011). Los procesos en desarrollo de integración de conocimiento científico integrado al local facilitan grupos de aprendizaje así como el apoyo a la generación de capacidades comunitarias para prepararse hacia la adaptación de la agricultura (Kettle et al., 2014).

Nuevos retos de investigación asociados a la variabilidad climática incluyen la generación de alternativas frente a la aparición de nuevas plagas y enfermedades, manifestación de otras ocasionales que se convierten en habituales, incremento de arvenses, reducción de reguladores naturales y disminución de la efectividad de métodos convencionales de control, etc. (Vázquez, 2011).

El indicador de planificación de la finca responde a la necesidad de regular las funciones ambientales y productivas del sistema optimizando el potencial que tiene y atendiendo a la necesidad de proteger áreas desgastadas o relictos ecosistémicos de importancia estratégica para la unidad productiva; pero al mismo tiempo, la planificación tiene el objetivo de responder oportunamente a la demanda del mercado coordinando las aspiraciones y posibilidades de cada socio de la ARAC para el mercadeo de sus productos.

El último indicador, mercadeo justo, refiere al fortalecimiento de la relación directa entre productores de la ARAC y consumidores de una red creada en Bogotá que adquiere los productos agroecológicos bajo la modalidad de canasta semanal con productos de cosecha. Para atender a esta demanda creciente y mantener el vínculo de confianza entre los productores y consumidores, la ARAC trabaja en la generación de un Sistema Participativo de Garantía (SGP) (Cuéllar \& Calle, 2009) que permita obviar la certificación de tercera parte a partir de un esquema de control basado en la confianza con participación de consumidores y productores. La estrategia no solo permite condiciones ventajosas para los productores que reciben mejores pagas por sus productos, sino también para los consumidores que reciben productos agroecológicos a precios justos y apoyan la gestión ambiental y social de la ARAC.

En términos de adaptabilidad, la necesidad de sostener este mercado regional motiva a una mejor planificación de la producción, ya que la falta de planificación colectiva de las fincas constituye una de las dificultades importantes para sostener esta demanda del mercado; pero al mismo tiempo, sostener la producción implica reducir los factores que atentan contra su estabilidad, lo que conduce a la implementación de estrategias tecnológicas que reduzcan la condición de vulnerabilidad del sistema. El indicador de mercado justo puede ser analizado también como un indicador de mitigación al cambio climático, en el sentido de que la relación de mercado directo entre productos y consumidores en un mercado local, reduce la emisión de gases generados por el transporte de alimentos a largas distancias.

En general, los valores promedios para los indicadores se incrementan en la totalidad de los indicadores entre la primera y segunda evaluación destacándose los indicadores semillas propias, estabilidad y fertilidad del suelo, investigación campesina, planificación de la finca, rentabilidad y mercadeo justo con diferencia estadísticamente significativa, concluyéndose que la implementación de las acciones para mejorar la sustentabilidad influyó directamente sobre los aspectos que mejoran la capacidad de las fincas para adaptarse a los riesgos de la variabilidad climática, especialmente a nivel de suelos, así como mejora en la competitividad de la agricultura, por el mejoramiento que se evidencia en la rentabilidad de la actividad productiva. 
Los indicadores de conservación de agua, arborización y participación en red, mostraron mejoras en los promedios globales para la totalidad de las fincas, pero estos aumentos no son aún consistentes en la totalidad de las mismas como para demostrar diferencia estadística, por lo que se requiere afianzar el seguimiento e implementación de prácticas para mejorar la adaptabilidad de los sistemas en estos indicadores precisos, sin descuidar los demás.

En la medida en que los riesgos detectados en la primera fase del estudio se relacionan de manera complementaria entre ellos aumentando la vulnerabilidad, en este mismo grado los indicadores se complementan unos a otros generando sinergias que mejoran la capacidad adaptativa y sustentabilidad del sistema productivo; por ejemplo, la conservación del agua y el mejoramiento en la estabilidad y fertilidad del suelo mejoran la producción, sostienen el mercado y generan mayor rentabilidad para el productor; la participación en red mejora en la planificación colectiva de las siembras y promueve la investigación local; esta, a su vez, mejora la producción y rentabilidad.

La mejora en los indicadores conduce a un mayor estado de adaptabilidad y sustentabilidad del sistema productivo disminuyendo su vulnerabilidad; esto, a nivel de finca, representa también una mejora importante en su desempeño global que se evidencia en los resultados de la evaluación de adaptabilidad y sustentabilidad para las fincas (Tabla 4). Los valores fueron obtenidos como promedio ponderado a partir de coeficientes para cada indicador construidos con participación de los agricultores, en el entendido de que no todos los indicadores tienen el mismo peso o incidencia sobre el grado de adaptabilidad o sustentabilidad global de la finca (Sarandón \& Flores, 2009).

Aunque todas las fincas evidencian mejora en los valores de los indicadores, se destacan las fincas El Tablón, Buena Vista, La Acacia y Almaguer con los mayores niveles de aumento de la adaptabilidad y sustentabilidad; los menores valores fueron para las fincas Serranías, Los Laureles y El Porvenir con mejoras inferiores a 0,5 en promedio y para las cuales se deben afinar los planes de mejoramiento. 
Tabla 4. Resultados de la evaluación de adaptabilidad y sustentabilidad de los sistemas productivos. Asociación Red Agroecológica Campesina de Subachoque.

\begin{tabular}{|l|c|c|c|}
\hline \multicolumn{1}{|c|}{ Finca } & $\begin{array}{c}\text { Promedio } \\
\text { ponderado } \\
\text { evaluación 1 } \\
(2013)\end{array}$ & $\begin{array}{c}\text { Promedio } \\
\text { ponderado } \\
\text { evaluación 2 } \\
(2014)\end{array}$ & Diferenda \\
\hline Buena Vista & 0,39 & 1,33 & 0,94 \\
\hline El Tablón & 0,44 & 1,50 & 1,06 \\
\hline La Acacia & $-0,33$ & 0,75 & 1,08 \\
\hline Almaguer & 0,22 & 1,22 & 1,00 \\
\hline Alcare & $-0,72$ & $-0,17$ & 0,55 \\
\hline El Porvenir & 0,56 & 0,89 & 0,33 \\
\hline San Luis & $-0,33$ & 0,50 & 0,83 \\
\hline Santa Otilia & 0,17 & 0,50 & 0,33 \\
\hline La Luz & 0,11 & 0,61 & 0,50 \\
\hline Campo Hermoso & $-0,72$ & 0,17 & 0,89 \\
\hline Los Laureles & 0,83 & 1,28 & 0,45 \\
\hline Serranías & 0,22 & 1,00 & 0,78 \\
\hline Las Hortensias & 0,22 & 1,06 & 0,84 \\
\hline
\end{tabular}

Fuente: los autores.

Los resultados de muchos estudios validan y realzan la importancia del trabajo agroecológico realizado por los campesinos, por su eficacia en la construcción de la resiliencia del agroecosistema (Henao, 2013). A nivel técnico la agroecología se basa en estrategias que estimulan la agrobiodiversidad, cierran los ciclos de nutrientes, promueven la autonomía de insumos utilizando recursos locales, conservan las características fisicoquímicas y biológicas de los suelos, reducen la erosión al emplear coberturas vivas, aprovechan las fuentes de energía renovable, integran ganaderíaagricultura y producción forestal, consumen menos combustibles fósiles, entre otras (Valdés \& Vargas, 2011; Márquez \& Funes-Monzote, 2013); a nivel sociocultural la agroecología propende por realzar el conocimiento local y tradicional, las relaciones basadas en la equidad de género, la participación y organización, la vinculación de toda la familia al sistema productivo democratizando las decisiones, etc. Por este conjunto de estrategias se corrobora que uno de los propósitos esenciales de la agroecología es justamente incidir sobre los procesos de cambio adaptativo en sistemas socioecológicos (Ríos-Osorio et al., 2013).

\section{Resiliencia, vulnerabilidad y adaptación}

Los resultados de la valoración de los indicadores en la ARAC demuestran cómo se han generado respuestas adaptativas para hacer frente a adversidades como la variabilidad climática reduciendo la vulnerabilidad. Altieri \& Nicholls (2013) resaltan cómo los agricultores por ellos mismos pueden incidir amentando su capacidad de reacción para desplegar mecanismos agroecológicos que mejoran su resiliencia y les permitan resistir y recuperarse de los eventos climáticos; tanto las estrategias técnicas como las sociales, constituyen elementos clave de la resiliencia. 
La resiliencia socioecológica constituye, entonces, un concepto apropiado para entender la sostenibilidad de un agroecosistema; a través de este se puede explicar la forma como colectivos humanos logran adaptar sus sistemas productivos a las características del entorno natural y a las aspiraciones socioculturales de la comunidad (Ríos-Osorio et al., 2013).

Salas-Zapata et al. (2012) diferencian dos respuestas adaptativas posibles en un sistema afectado por una perturbación fuerte: la adaptación y la transformación. En el primer caso el sistema se reorganiza conservando los procesos esenciales y las interacciones entre sus componentes, así como sus mecanismos de control [Holling (1973), Kinzig et al. (2006) y Walker et al. (2006) citados en Salas-Zapata et al. (2012)]; en el segundo cambian su estructura, funciones, interacciones y mecanismos de control. La adaptación por la vía de estrategias agroecológicas restablece interacciones naturales planta-animal, suelo-microorganismos, predador-presa, microrganismo-planta, agricultor-cultivo; cultura-paisaje, finca-comunidad, etc., estabilizando el funcionamiento global del sistema. Esta capacidad de reajustarse adaptativamente a las dinámicas del entorno social y ecológico es la que ha permitido a los productores agroecológicos satisfacer sus necesidades y mejorar sus condiciones de vida; condición propia de los agroecosistemas sustentables.

Los campesinos han sido capaces históricamente de afrontar, resistir y recuperarse de fenómenos externos que atentan contra la estabilidad de sus sistemas productivos incluidos eventos climáticos extremos; las fincas agroecológicas evidencian un enorme potencial para aumentar la resiliencia frente a adversidades; potencial que debe ser reconocido y promovido localmente en términos de políticas públicas.

\section{CONCLUSIONES}

La construcción participativa de marcos de análisis de riesgos frente a la variabilidad climática a partir de un enfoque multidimensional, permite establecer indicadores locales consistentes con la situación real, empoderando a los actores locales para reconocer su grado de vulnerabilidad e implementar estrategias de adaptación de sus sistemas productivos. Mientras los riesgos para el sistema productivo se relacionan de manera complementaria aumentando la vulnerabilidad, los indicadores de sustentabilidad se complementan unos a otros de manera sinérgica mejorando su capacidad adaptativa.

La aplicación de un enfoque adaptativo centrado en la comunidad manifestado en la construcción y valoración de los indicadores, incrementó los niveles de sustentabilidad y adaptabilidad, de forma que la implementación de las acciones de mejora influyó positivamente sobre la capacidad de los sistemas socioecológicos para adaptarse a los riesgos de la variabilidad climática.

Las estrategias agroecológicas implementadas como acciones de mejora, incrementaron la resiliencia socioecológica de los agroecosistemas manejados por agricultores familiares asociados a la Red Agroecológica Campesina de Subachoque (Cundinamarca), aumentando la posibilidad de que respondan mejor ante cualquier escenario de cambio ambiental futuro. 


\section{AGRADECIMIENTOS}

A la Facultad de Ingeniería y a la División de Investigaciones de UNIMINUTO, por el apoyo recibido para el desarrollo de este trabajo. A los miembros de la Asociación Red Agroecológica Campesina por su disposición para el trabajo cooperativo.

\section{POTENCIAL CONFLICTO DE INTERESES}

Los autores manifiestan que no existe conflicto de intereses alguno respecto al presente artículo.

\section{FUENTES DE FINANCIACIÓN}

El proyecto de investigación fue financiado por la División de Investigaciones del Sistema Universitario UNIMINUTO, Bogotá.

\section{REFERENCIAS}

- Acevedo, Á., \& Angarita, A. (2013). Metodología para la evaluación de sustentabilidad a partir de indicadores locales para el diseño y desarrollo de programas agroecológicos. MESILPA. Bogotá: UNIMINUTO.

- Altieri, M. A. (1995). Bases científicas de la agroecología. Santiago de Chile: CETAL.

- Altieri, M. A. (2013). Construyendo resiliencia socio-ecológica en agroecosistemas: algunas consideraciones conceptuales y metodológicas. En C. Nicholls, L. A. Ríos, M. A. Altieri (Eds.), Agroecología y resiliencia socioecológica: adaptándose al cambio climático (pp. 94-104). Medellín: SOCLA, REDAGRES.

- Altieri, M. A., \& Nicholls, C. I. (2007). Biodiversidad y manejo de plagas en agroecosistemas. Barcelona: Icaria Editorial.

- Altieri, M. A., \& Nicholls, C. I. (2013). Agroecología y resiliencia al cambio climático: principios y consideraciones metodológicas. Revista de Agroecología, 8(1), 7-20. 
- Anderies, J. M., Janssen, M. A., \& Ostrom, E. (2004). A framework to analyze the robustness of social-ecological systems from an institutional perspective. Ecol. Soc., 9(1), 18. Recuperado de http://www.ecologyandsociety.org/vol9/iss1/art18/

- Boshell, J. F. (2010). Contextualización de los efectos del cambio climático en la agricultura. Lima: InWent.

- Conway, G. R. (1994). Sustainability in agricultural development: trade-offs between productivity, stability and equitability. Journal of Farming Systems and ResearchExtensions, 4(2), 1-14

- Crane, T. A., Roncoli, C., \& Hoogenboom, G. (2011). Adaptation to climate change and climate variability: the importance of understanding agriculture as performance. Journal of Life Sciences, 57, 179-185.

- Cuéllar, M., \& Calle, Á. (2009). Sistemas participativos de garantía: poder, democracia y agroecología. En I Congreso de Sociología de la Alimentación. Barcelona, España.

- Forero, J. (2010). El campesino colombiano: entre el protagonismo económico y el desconocimiento de la sociedad. Bogotá: Facultad de Estudios Ambientales y Rurales, Pontifica Universidad Javeriana.

- Gliessman, S. (2002). Agroecología. Procesos ecológicos en agricultura sustentable. Turrialba, Costa Rica: CATIE.

- Guzmán, G., González, M., \& Sevilla, E. (2000). Introducción a la agroecología como desarrollo rural sostenible. Madrid: Ediciones Mundi-Prensa.

- Henao, A. (2013). Propuesta metodológica de medición de la resiliencia agroecológica en sistemas socio-ecológicos: un estudio de caso en los andes colombianos. Revista de Agroecología, 8(1), 85-91.

- Hernández, A., Vargas, D., Borges, Y., Ríos, H., Morales, M., \& Funes-Monzote, F. R. (2011). Reservas de carbono orgánico en suelos ferralíticos rojos. En H. Ríos, D. Vargas, F. R. Funes-Monzote (Comps.), Innovación agroecológica, adaptación y mitigación del cambio climático (pp. 45-55). La Habana: Instituto Nacional de Ciencias Agrícolas.

- Holdrige, L. R. (1987). Ecología basada en zonas de vida. San José, Costa Rica: Instituto Interamericano de Cooperación para la Agricultura. 
- Hünnemeyer, A.-J., De Camino, R., \& Müller, S. (1997). Análisis del desarrollo sostenible en Centroamérica: indicadores para la agricultura y los recursos naturales.San José, Costa Rica: IICA/GTZ.

- $\quad$ IPCC -Intergovernmental Panel on Climate Change-. (2007). Cambio climático 2007. Informe de síntesis. Recuperado de http://www.ipcc.ch/pdf/assessmentreport/ar4/syr/ar4_syr_sp.pdf

- Kettle, N. P., Dow, K., Tuler, S., Webler, T., Whitehead, J., \& Miller, K. M. (2014). Integrating scientific and local knowledge to inform risk-based management approaches for climate adaptation. Climate Risk Management, 4-5, 17-31.

- Kumaraswamy, S. (2012). Sustainability issues in agro-ecology: Socio-ecological perspective. Agricultural Sciences, 3(2), 153-169.

- Lahsen, M., Sánchez-Rodríguez, R., Romero, P., Dube, P., Leemans, R., Gaffney, O.,...Smith, M. S. (2010). Impacts, adaptation and vulnerability to global environmental change: challenges and pathways for an action-oriented research agenda for middleincome and low-income countries. Current Opinion in Environmental Sustainability, 2, 364-374.

- Lovell, S. T., DeSantis, S., Nathan, C. A., Breton, M., Méndez, E., Kominami, H. C.,... Morris, W. (2010). Integrating agroecology and landscape multifunctionality in Vermont: An evolving framework to evaluate the design of agroecosystems. Agricultural Systems, 103, 327-341.

- Márquez, M., \& Funes-Monzote, F. R. (2013). Factores ecológicos y sociales que explican la resiliencia al cambio climático de los sistemas agrícolas en el municipio de La Palma, Pinar del Río, Cuba. Revista de Agroecología, 8(1), 43-52.

- Masera, O., Astier, M., \& López-Ridaura, S. (1999). Sustentabilidad y manejo de recursos naturales; el marco de evaluación MESMIS. México: Grupo Interdisciplinario de Tecnología Rural Apropiada.

- Meinke, H., Howden, S. M., Struik, P., Nelson, R., Rodríguez, D., \& Chapman, S. (2009).Adaptation science for agriculture and natural resource management - urgency and theoretical basis. Current Opinion in Environmental Sustainability, 1, 69-76.

- Miranda, T., Sánchez, S., Sánchez, T., Lamela, L., \& Álvarez, D. (2011). Innovación local participativa ante el cambio climático. En H. Ríos, D. Vargas, F. R. Funes- 
Monzote (Comps.), Innovación agroecológica, adaptación y mitigación del cambio climático(pp. 213-220). La Habana: Instituto Nacional de Ciencias Agrícolas.

- Misra, A. K. (2014). Climate change and challenges of water and food security.International Journal of Sustainable Built Environment, 3, 153-165.

- Molinet, Y., Álvarez, A., González, P. J., Damas, R., \& Ruiz, R. (2011). Descontaminación de residuales, producción de biomasa y energía y reciclaje de nutrientes. En H. Ríos, D. Vargas, F. R. Funes-Monzote (Comps.), Innovación agroecológica, adaptación y mitigación del cambio climático (pp. 55-74). La Habana: Instituto Nacional de Ciencias Agrícolas.

- PNUD Colombia. (2011). Informe Nacional de Desarrollo Humano. Colombia rural: razones para la esperanza. Bogotá: Programa de las Naciones Unidas para el Desarrollo.

- Ríos, H., Miranda, S., \& Vargas, D. (2011). Sistemas descentralizados y participativos para la adaptación y mitigación del cambio climático en Cuba. En H. Ríos, D. Vargas, F. R. Funes-Monzote (Comps.), Innovación agroecológica, adaptación y mitigación del cambio climático (pp. 3-11). La Habana: Instituto Nacional de Ciencias Agrícolas.

- Ríos-Osorio, L. A., Salas-Zapata, W., \& Espinosa-Alzate, J. A. (2013). Resiliencia socioecológica de los agroecosistemas, más que una externalidad. En C. Nicholls, L. A. Ríos, M. A. Altieri (Eds.), Agroecología y resiliencia socioecológica, adaptándose al cambio climático (pp. 64-76). Medellín: SOCLA, REDAGRES.

- Rogé, P., \& Astier, M. (2013). Previniéndose para el cambio climático: una metodología participativa. En C. Nicholls, L. A. Ríos, M. A. Altieri (Eds.), Agroecología y resiliencia socioecológica, adaptándose al cambio climático (pp. 124-148). Medellín: SOCLA, REDAGRES.

- Salas-Zapata, W. A., Ríos-Osorio, L. A., \& Álvarez-Del Castillo, J. (2012). Marco conceptual para entender la sustentabilidad de los sistemas socioecológicos. Ecología Austral, 22(1). Recuperado de http://www.scielo.org.ar/scielo.php?pid=S1667782X2012000100008\&script=sci_arttext

- Sarandón, S. J., \& Flores, G. (2009). Evaluación de la sustentabilidad en agroecosistemas: una propuesta metodológica. Revista Agroecología, 4, 19-28. 
- Seaman, J. A., Sawdon, G. E., Acidri, J., \& Petty, C. (2014). The household economy Approach. Managing the impact of climate change on poverty and food security in developing countries. Climate Risk Management, 4-5, 59-68.

- Seidl, G. U., Morales, H., Arriola, L. A., \& Evangelista, A. A. (2011). Ya no hay árboles ni agua. Perspectivas de los cambios ambientales en comunidades de Zinacatán, Chiapas.LiminaR. Estudios Sociales y Humanísticos, IX(1), 97-119.

- Sevilla-Guzmán, E. (2006). De la sociología rural a la agroecología. Barcelona: Icaria Editorial.

- Smyth, A. J., \& Dumanski, J. (Eds.). (1993). FESLM: an international framework for evaluating sustainable land management. Roma: FAO.

- Valdés, N., \& Vargas, D. (2011). Gases efecto de invernadero en la agricultura, un llamado a la acción. En H. Ríos, D. Vargas, F. R. Funes-Monzote (Comps.), Innovación agroecológica, adaptación y mitigación del cambio climático (pp. 15-23). La Habana: Instituto Nacional de Ciencias Agrícolas.

- Vázquez, L. L. (2011). Cambio climático, incidencia de plagas y prácticas agroecológicas resilientes. En H. Ríos, D. Vargas, F. R. Funes-Monzote (Comps.), Innovación agroecológica, adaptación y mitigación del cambio climático (pp. 75-101). La Habana: Instituto Nacional de Ciencias Agrícolas.

- Zimmerer, K. (2010). Biological diversity in agriculture and global change. The Annual Review of Environment and Resources, 3, 137-66.

1. Investigación desarrollada en el marco del proyecto: Promoción de estrategias agroecológicas para mejorar la resiliencia de la agricultura altoandina frente al cambio climático en el municipio de Subachoque - Cundinamarca - C113-74, con el apoyo de la División de Investigaciones del Sistema Universitario UNIMINUTO.

2. PhD Agroecología. Máster en Agroecología y Desarrollo Rural Sostenible. Profesor e investigador del programa Ingeniería Agroecológica, Corporación Universitaria Minuto de Dios, UNIMINUTO. Bogotá, Colombia. alacevedo@uniminuto.edu

3. Magíster en Educación. Profesor e investigador del programa Ingeniería Agroecológica, Corporación Universitaria Minuto de Dios, UNIMINUTO. Bogotá, Colombia.aangarita@uniminuto.edu

4. Ingeniera en Agroecología. Corporación Universitaria Minuto de Dios, UNIMINUTO. Bogotá, Colombia. juanitafquiroga@gmail.com

5. Ingeniera en Agroecología. Corporación Universitaria Minuto de Dios, UNIMINUTO. Bogotá, Colombia.viviagro10@gmail.com 
Para citar este artículo: Acevedo-Osorio, Á., Angarita Leiton, A., León Durán, M. V., \& Franco Quiroga, K. L. (2017). Sustentabilidad y variabilidad climática: acciones agroecológicas participativas de adaptación y resiliencia socioecológica en la región alto-andina colombiana. Luna Azul, 44, 06-26. DOI: 10.17151/luaz.2017.44.2. Recuperado de http://200.21.104.25/lunazul/index.php?option=com_content\&view=article\&id=217

Esta obra está bajo una Licencia de Creative Commons Reconocimiento CC BY

(). (1)

